\title{
Human Brucellosis Caused by Brucella canis: A Rare Case Report
}

\author{
Mohammad Mahdi Majzoobi ${ }^{1}$, Hamid Reza Ghasemi Basir ${ }^{2}$, Mohammad Reza Arabestani ${ }^{3}$, Surur \\ Akbari $^{1, *}$ and Haleh Nazeri ${ }^{2}$ \\ ${ }^{1}$ Brucellosis Research Centre, Hamadan University of Medical Sciences, Hamadan, Iran \\ ${ }^{2}$ Department of Pathology, Hamadan University of Medical Sciences, Hamadan, Iran \\ ${ }^{3}$ Department of Microbiology, Hamadan University of Medical Sciences, Hamadan, Iran \\ "Corresponding author: Brucellosis Research Centre, Sina Hospital, Mirzadeh Eshghi Ave., Hamadan, Iran. Tel: +98-8138274184, Email: suak1393@gmail.com
}

Received 2017 October 11; Revised 2018 February 15; Accepted 2018 June 26.

\begin{abstract}
Introduction: Brucella canis, a member of Brucella species, has been reported as a cause of human brucellosis in a few cases, and routine serologic tests do not identify this species. In spite the fact that Iran is an endemic area for brucellosis, there has not been any report of human brucellosis due to $B$. canis.

Case Presentation: The patient was a 68-year-old female with complaints of fever, foul smelling urine, malaise, vomiting, and arthralgia. Considering significant leucocytosis and pyuria, positive urine culture antibiotic therapy was initiated for urosepsis. However, due to non-suitable response to antibiotic and negative serologic study for brucellosis, supplemental assessments, including bone marrow aspiration and biopsy (BMA\&B), were performed to study malignancy, tuberculosis, and brucellosis. Brucella canis was isolated from BACTEC media and subsequently confirmed by polymerase chain reaction test.

Conclusions: Considering the ineffectiveness of routine serologic study for diagnosis of $B$. canis in the presence of clinical presentations suggestive for brucellosis, blood or BM culture should be considered.
\end{abstract}

Keywords: Brucellosis, Brucella canis, Iran

\section{Introduction}

Brucella canis, first identified in the late 1960s (1), was reported in individuals with close contact to infected dogs and in laboratorians working with cultured B. canis (2). Symptoms and Signs of patients are generally similar to, yet milder than those of brucellosis caused by B. abortus and B. melitensis (3).

Since routine serologic diagnosis of brucellosis does not include $B$. canis, infection with this species may be more widespread than currently suspected (4).

The gold standard diagnostic test remains the culture and isolation of coccobacilli from a clinical specimen $(4,5)$. polymerase chain reaction (PCR) assays are able to discriminate various species of Brucella, including B. canis and vaccine strains $(6,7)$.

Iran is one of the major endemic regions for brucellosis with high incidence of brucellosis among human and animal populations. The most common species is B. Melitansis and $B$. abortus, and until the present, there has not been any report of human brucellosis due to B. canis in $\operatorname{Iran}(8,9)$. The aim of this paper was to report a case of human brucellosis, caused by B. canis.

\section{Case Presentation}

The patient was a 68-year-old female, an inhabitant of the rural area of Hamadan province, located in the west of Iran. She was referred to the centre with complaints of fever and chills, foul smelling urine, malaise, nausea and vomiting, lower back pain, and right knee arthralgia for the previous three weeks. She kept a few sheeps at her home and consumed native dairy products. Physical examination revealed ill looking and lethargic elderly females with body temperature of $39.8{ }^{\circ} \mathrm{C}$, PR: 96/minute, RR:18/minute and BP:130/70 mmHg. There were no abnormal findings in head and neck, chest, abdomen, and neurologic examination. All joints, including both sacroiliacs and knees had normal range of motion, without any tenderness or effusion.

Results of laboratory tests made on admission were as follow: significant leukocytosis, pyuria, positive urine culture for Escherichia coli with colony count of $100000 / \mathrm{mL}$, $\mathrm{BUN}=35, \mathrm{Cr}=2.1, \mathrm{ESR}=11, \mathrm{CRP}=+3$, and negative Wright and $2 \mathrm{ME}$. Abdominopelvic sonography revealed spleen in the upper limit of the normal size. According to diagnosis of urosepsis, treatment was initiated by ceftriaxone. Although UA was normalized and UC was negative on the 
fifth day, no improvements were obvious in symptoms and signs of the patient's fever, weakness, and generalized pain. Therefore, as a case of fever with unknown origin (FUO), whole body scans, in addition to serologic tests of brucellosis were requested for a second time. Whole body scan exhibited degenerative changes in lumbar spine and knee joints, and result of the second serologic study was negative again; therefore, bone marrow aspiration and biopsy (BMA\&B) were taken to study malignancies, tuberculosis, and brucellosis in spite of negative serology. After five days, the culture of BM in BACTEC media was reported positive for Brucella sp., and sub-culture on EMB and blood agar media revealed small mucoid, slightly yellowish translucent non-haemolytic convex colony composed of small gram negative coccobacilli arranged in pairs or as single cells in microscopic examination. This gram negative fastidious bacillus was oxidative on OF media without motility on SIM media and wet smear. The biochemical tests signified $\mathrm{H}_{2} \mathrm{~S}$ negative, oxidase positive, and catalase positive micro-organism that hydrolysed urea in less than five minutes. The negative serology, mucoid colony, and biochemical characteristics were indicative of B. canis.

Microscopic examination of bone marrow tissue revealed no evidence of lymphoproliferative, granulomatous or metastatic lesions. The patient showed favourable clinical response to treatment with ciprofloxacin and doxycycline within a week. In addition to fever cessation, her musculoskeletal pain decreased significantly and Serum creatinine normalized.

For confirming identified B. canis, the specific PCR with primer sequences (ITS66: ACATAGATCGCAGGCCAGTCA and ITS279: AGATACCGACGCAAACGCTAC)(7) and sequencing of PCR product were done. The results of PCR and sequencing confirmed B. canis.

\section{Discussion}

Iran is an endemic area and Hamadan is among very high incidence regions for brucellosis (8). According to the annual report of Iranian CDC, the most common causes of human brucellosis are B. melitensis and B. abortus, with no report of $B$. canis or B. Suis. However, several studies have revealed that $B$. canis seroprevalence rate among the dogs population ranges from $3.5 \%$ to $41.2 \%$ in different regions of Iran (10-12). Since 1973, the CDC of America has isolated $B$. canis from approximately 50 human specimens. Low numbers of identified human $B$. canis cases indicates that this Brucella sp. is probably not a significant public health concern; in addition, B. canis could be under-diagnosed or under-reported due to nonspecific presentation of the disease and lack of available laboratory testing (13). One of the transmission routes of $B$. canis is close contact to infected dogs. Since the patient resided in a village, which has many stray dogs, she might have been at risk of acquiring B. canis from dogs $(1,3)$.

Although there are multiple studies that state B. canis infections tend to cause milder illness compared to other Brucella sp., some studies have shown serious manifestations caused by B. canis $(13,14)$. Problem list of the current patient, also, demonstrates that her disease severity was not mild.

In the absence of adequate culture, which facilitates isolation of Brucella sp., the diagnosis of brucellosis depends on serological tests, yet available serologic tests are ineffective in diagnosing $B$. canis infections $(4,7)$. Therefore, results of serological assay were negative in the current patient.

The standard diagnostic method remains to be the isolation of Brucella from blood cultures or host tissues (15). In contrast to other Brucella species, which grow in smooth colonies, $B$. canis naturally forms rough phase (mucoid) colonies in culture. The appearance of colonies in bone marrow culture of the current case was also mucoid. Brucella canis has negative agglutination test, and does not produce $\mathrm{H}_{2} \mathrm{~S}$ with urease activity of less than a half hour. In addition, it grows in thionine yet not in fushin media. According to rapid urease test, negative results of the patient Wright test and fastidious bacilli grown in bone marrow culture demonstrate possibility of unknown Brucella sp. including $B$. suis and $B$. canis (16). Absence of $\mathrm{H}_{2}$ S production was more compatible with $B$. canis yet not sufficient enough to exclude $B$. suis. In this patient, existence of negative serology, biochemical characteristics, and mucoidal appearance of colonies specified B. canis.

Molecular techniques could be used for diagnosis of human brucellosis. The polymerase chain reaction (PCR) appears to offer several advantages over conventional methods: It is easy to perform, rapid, and safe for laboratory staff because serum-based PCR-assay will reduce the risk of handling the microorganism $(6,15)$. In this study, specific PCR is used for diagnosis and confirmation of $B$. $c a-$ nis, which was reported by biochemical and culture methods. In addition, sequencing methods were used for confirming the PCR product.

\subsection{Conclusion}

In case of low value of available serologic tests for diagnosis of $B$. canis, if the serologic tests are negative in patients suspected of having brucellosis, it is suggested to do blood or BM culture and serology concomitantly. 


\section{Footnote}

Conflict of Interests: The authors declare no conflict of interest.

\section{References}

1. Lucero NE, Corazza R, Almuzara MN, Reynes E, Escobar GI, Boeri E, et al Human Brucella canis outbreak linked to infection in dogs. Epidemiol Infect. 2010;138(2):280-5. doi: 10.1017/S0950268809990525. [PubMed: 19653929].

2. Wallach JC, Giambartolomei GH, Baldi PC, Fossati CA. Human infection with M- strain of Brucella canis. Emerg Infect Dis. 2004;10(1):1468. doi: 10.3201/eid1001.020622. [PubMed: 15078613]. [PubMed Central: PMC3322762].

3. Scheftel J. Brucella canis: Potential for zoonotic transmission. Compend Contin Educ Pract Vet. 2003;25(11):846-52.

4. Lucero NE, Escobar GI, Ayala SM, Jacob NO. Diagnosis of human brucellosis caused by Brucella canis. J Med Microbiol. 2005;54:457-61. doi: 10.1099/jmm.0.45927-0. [PubMed: 15824423].

5. Young EJ. An overview of human brucellosis. Clin Infect Dis. 1995;21(2):283-9. quiz 290. [PubMed: 8562733].

6. Garcia-Yoldi D, Marin CM, de Miguel MJ, Munoz PM, Vizmanos JL, Lopez-Goni I. Multiplex PCR assay for the identification and differentiation of all Brucella species and the vaccine strains Brucella abortus S19 and RB51 and Brucella melitensis Rev1. Clin Chem. 2006;52(4):77981. doi: 10.1373/clinchem.2005.062596. [PubMed: 16595839].

7. Keid LB, Soares RM, Vieira NR, Megid J, Salgado VR, Vasconcellos SA, et al. Diagnosis of canine brucellosis: comparison between serological and microbiological tests and a PCR based on primers to 16S-23S rDNA interspacer. Vet Res Commun. 2007;31(8):951-65. doi: 10.1007/s11259006-0109-6. [PubMed: 17308888].
8. Hashemi SH, Gachkar L, Keramat F, Mamani M, Hajilooi M, Janbakhsh A, et al. Comparison of doxycycline-streptomycin, doxycyclinerifampin, and ofloxacin-rifampin in the treatment of brucellosis: a randomized clinical trial. Int J Infect Dis. 2012;16(4):e247-51. doi: 10.1016/j.ijid.2011.12.003. [PubMed: 22296864].

9. Center for Disease Control and Prevention. Annual brucellosis registry report. Vice Chancellor of Health Deputy of Hamadan University of Medical Sciences, Hamadan, Iran; 2014.

10. Esmaeili H. Brucellosis in Islamic Republic of Iran. J Med Bacteriol. 2014;3(3-4):47-57.

11. Akhtardanesh B, Ghanbarpour R, Babaei H, Nazeri M. Serological evidences of canine brucellosis as a new emerging disease in Iran. Asian Pac J Trop Dis. 2011;1(3):177-80. doi: 10.1016/s2222-1808(11)60023-6.

12. Bigdeli M, Namavari MM, Moazeni-Ju F, Sadeghzade S, Mirzaei A. First study prevalence of brucellosis in Stray and Herding dogs South of Iran. Asian J Anim Vet Adv. 2011;10(10):1322-6. doi: 10.3923/javaa.2011.1322.1326.

13. Kazmierczak J. Public health implications of Brucella canis infections in humans. Summary findings and recommendations of the brucella canis workgroup. National Association of State Public Health Veterinarians; 2012.

14. Lucero NE, Jacob NO, Ayala SM, Escobar GI, Tuccillo P, Jacques I. Unusual clinical presentation of brucellosis caused by Brucella canis. J Med Microbiol. 2005;54(Pt 5):505-8. doi: 10.1099/jmm.0.45928-0. [PubMed: 15824432].

15. Garshasbi M, Ramazani A, Sorouri R, Javani S, Moradi S. Molecular detection of Brucella species in patients suspicious of Brucellosis from Zanjan, Iran. Braz J Microbiol. 2014;45(2):533-8. [PubMed: 25242938]. [PubMed Central: PMC4166279].

16. Alton GG, Forsyth JRL. Brucella. In: Baron S, editor. Medical microbiology. 4th ed. Galveston; 1996. p. 429-41. 